Ann. Biol. anim. Bioch. Biophys., 1977, 17 (4), 603-606.

\title{
Influence de la motricité intestinale sur l'absorption de l'eau et du glucose. Etude sur le duodénum irrigué in situ chez le rat anesthésié.
}

\author{
par M. LEGOUX, M. C. LEMAIRE, J. THOUVENOT \\ Laboratoire de Physiologie, Foculté de Médecine, Université de Tours, \\ 2 bis bd Tonnellé, 37032 Tours Cedex.
}

Summary. Influence of intestinal motility on water and glucose absorption in the anaesthetized rat.

The influence of intestinal motility on water and glucose absorption was investigated in the anaesthetized rat (urethane $1 \mathrm{~g} \cdot \mathrm{kg}^{-1}$ ). Infusion of Tyrode solution with glucose

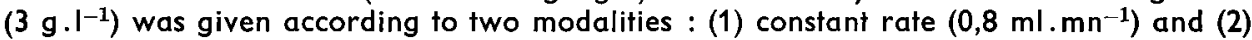
spontaneous rate with constant pressure of input $(7 \mathrm{~cm}$ water $)$. Comparison of the results obtained in both conditions shows that the ratio - amount of absorbed substance/total quantity introduced into the lumen was greater than that obtained in free-rate perfusion (water $50 \pm 8$ p. 100, glucose : $49 \pm 10$ p. 100).

The present results emphasize the influence of mechanical conditions for optimisation of intestinal absorption.

Le problème que nous nous sommes posé, est de savoir si, par le jeu de sa motricité, une anse intestinale « in vivo » est capable de modifier ses conditions d'absorption, notamment son débit d'irrigation, en vue d'optimiser l'absorption de l'eau et du glucose.

Des travaux déjà effectués au Laboratoire (Thouvenot et Rougereau, 1964) nous montraient que l'irrigation d'une anse par du glucose modifiait le tonus et l'activité contractile de celle-ci, et on avait constaté par ailleurs que les caractéristiques viscoélastiques changeaient selon que l'animal était à jeun ou non (Rougereau ef Thouvenot, 1965 ; Thouvenot, Rougereau et Frette, 1966).

En vue d'étudier le rôle joué par cette modification de motricité sur le bilan quantitatif de l'absorption, nous nous sommes proposé de comparer deux séries d'expériences : dans l'une, série témoin I, le débit d'irrigation est imposé (motricité exclue), dans l'autre, série II, le débit dépend entièrement de la motricité.

\section{Technique.}

Les essais sont pratiqués sur 31 rats, mâles, indemnes de toute affection pathogène, de 350 à $400 \mathrm{~g}$, à jeun depuis $16 \mathrm{~h}$, anesthésiés à l'uréthane intrapéritonéal $\left(1 \mathrm{~g} \cdot \mathrm{kg}^{-1}\right)$. 
Après laparotomie médiane de $6 \mathrm{~cm}$ environ, on insère une canule orale au niveau du rumen. Cette canule est poussée doucement dans l'estomac puis le duodénum. Ce procédé permel d'exclure le jeu de la régulation du transit par le système antropylorique. La canule orale liée au rumen est branchée sur un tube en $T$ qui permet la connection avec un capteur de pression. Une deuxième canule de diamètre $4 \mathrm{~mm}$ est fixée de 10 à $20 \mathrm{~cm}$ du pylore suivant les expériences. La préparation ainsi réalisée est reliée à deux systèmes d'irrigation :

- Série 1: Un débit de $0,8 \mathrm{ml} \cdot \mathrm{mn}^{-1}$ de Tyrode glucosé $\left(3 \mathrm{~g} \cdot \mathrm{I}^{-1}\right)$ est imposé par une pompe péristaltique à l'entrée de la canule orale.

- Série II: La canule orale est reliée à un réservoir de Tyrode glucosé (a), placé à une hauteur constante de $7 \mathrm{~cm}$ au-dessus de la préparation. La seule contrainte subie par l'intestin esł cette pression d'entrée choisie comme pression optimale favorisant la motricité (de Lavernhe-Lemaire, 1976).

Par le jeu de la motricité intestinale, le liquide est donc prélevé dans le réservoir de Tyrode (a) où le niveau est maintenu constant par le système d'asservissement suivant (fig. 1). L'alimentation A est dirigée soit dans le récipient (a), soit dans le récipient

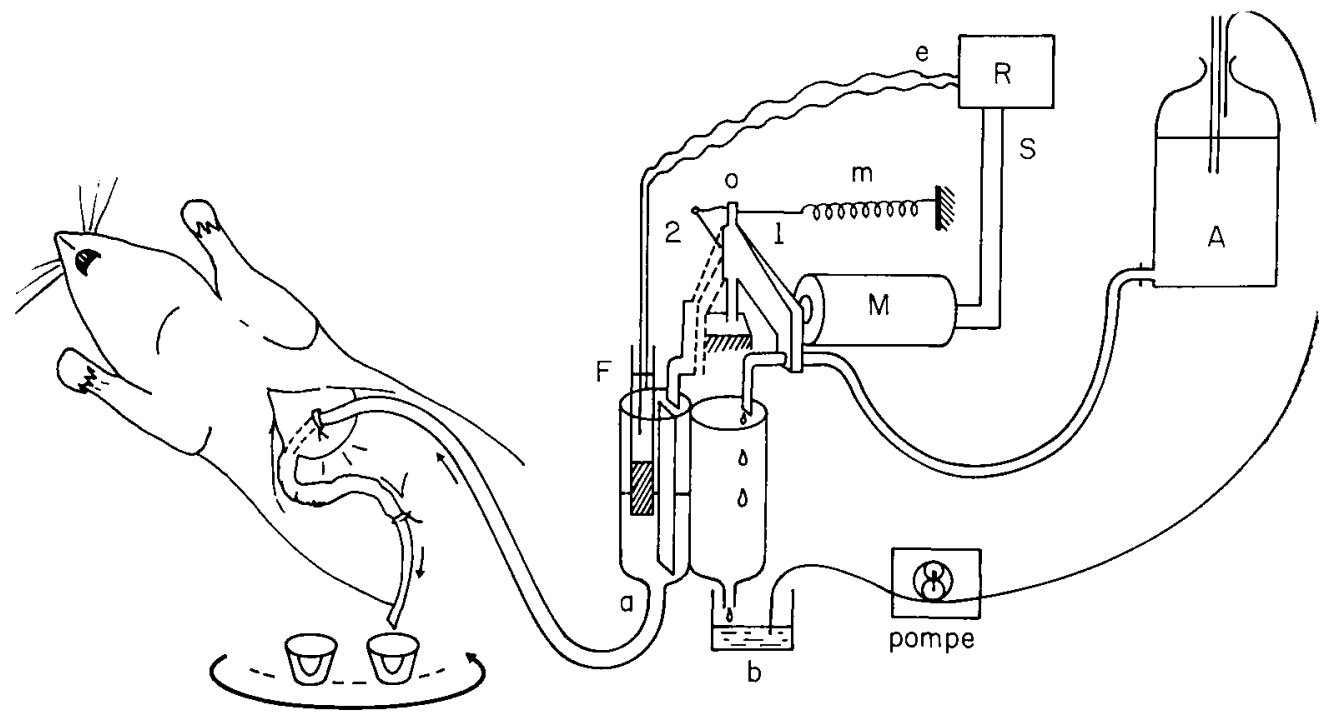

FIG. 1. Schéma du dispositif d'irrigation d'une anse duodénale in vivo.

A droite, système d'irrigation à débit asservi : $a$, récipient à niveau constant avec flotteur $F$ muni de contacts, $R$, relais ; dispositif électromécanique $M$ permettant l'apport de liquide d'irrigation provenant du récipient $A$, soit vers a relié à l'intestin, soit vers le système de récupération b. (Pour le fonctionnement, voir texte).

(b) selon la position d'un bras mû par un électro-aimant $(M)$. Celui-ci dirige l'alimentation vers (b), lorsque la montée du flotteur (F) situé dans le récipient (a) met en jeu le relais $(R)$. Chaque impulsion du relais est enregistrée, ce qui permet de calculer le débit d'entrée à chaque instant. 
Pour les deux systèmes, le liquide évacué par la canule aborale est recueilli dans un collecteur de fractions. On dispose des données suivantes : - débit d'entrée en $\mathrm{ml} \cdot \mathrm{mn}^{-1}$; - débit de sortie en $\mathrm{ml} \mathrm{mn}^{-1}$; - concentration en glucose du liquide d'entrée en $\mathrm{g} \cdot \mathrm{I}^{-1} ;-$ concentration en glucose du liquide de sortie en $\mathrm{g} \cdot \mathrm{I}^{-1}$.

On peut calculer l'absorption relative, c'est-à-dire la quantité de substance absorbée rapportée à la quantité introduite dans l'anse pendant un temps donné.

\section{Résultats.}

\section{Absorption.}

Les résultats brułs (tabl. I), malgré leur dispersion, font apparaître que l'absorption de l'eau et du glucose est beaucoup plus élevée en débit libre qu'en débit imposé.

TABLEAU 1

Comparaison des débits d'absorption d'eau et de glucose en débit imposé et en débit libre

\begin{tabular}{|c|c|c|c|c|c|c|c|}
\hline & & & Eau & & & Glucose & \\
\hline & $\mathbf{N}$ & $\begin{array}{l}\text { Absorption } \\
\mathrm{ml} / \mathrm{h} / \mathrm{cm}\end{array}$ & Extrêmes & $\begin{array}{l}\text { Absorpfion } \\
\text { relative }\end{array}$ & $\begin{array}{c}\text { Absorption } \\
\mathrm{mg} / \mathrm{h} / \mathrm{cm}\end{array}$ & Extrêmes & $\begin{array}{l}\text { Absorption } \\
\text { relative }\end{array}$ \\
\hline Débit imposé & 21 & 0,54 & $\begin{array}{l}0 \\
1,25\end{array}$ & $0,12 \pm 0,04$ & 4,26 & $\begin{array}{l}0,85 \\
6,57\end{array}$ & $0,30 \pm 0,04$ \\
\hline Test $t \ldots \ldots$ & & & $\mathrm{DL}=29$ & $\begin{array}{l}t=14,2 \\
P<0,001\end{array}$ & & $\mathrm{DL}=29$ & $\begin{array}{l}t=5,7 \\
P<0,001\end{array}$ \\
\hline Débit libre .. & 10 & 3,93 & $\begin{array}{l}1,3 \\
6,3\end{array}$ & $0,50 \pm 0,08$ & 13,6 & $\begin{array}{r}9,7 \\
18,7\end{array}$ & $0,49 \pm 0,10$ \\
\hline
\end{tabular}

Le test † a été appliqué à l'absorption relative soit de l'eau, soit du glucose.

En revanche, le calcul de l'absorption relative (quantité absorbée sur quantité d'entrée), montre une différence très significative entre ces deux situations expérimentales $(P<0,001)$. Ce rapport est plus grand en débit libre $(50 \pm 10$ p. 100 pour le glucose) qu'en débit imposé $(15 \pm 4$ p. 100 pour l'eau et $29 \pm 4$ p. 100 pour le glucose).

Ce pourcentage d'absorption est donc plus élevé en débit libre, bien que le débit d'irrigation soit plus important :

- $0,8 \mathrm{ml} \cdot \mathrm{mn}^{-1}$ en débit imposé ;

- en débit libre, le débit de liquide prélevé par l'intestin est modifié selon la longueur d'anse perfusée (ex. : $1,5 \mathrm{ml} . \mathrm{mn}^{-1}$ pour une perfusion de moins de $15 \mathrm{~cm}$ et $3 \mathrm{ml} . \mathrm{mn}^{-1}$ pour une perfusion d'une anse de $20 \mathrm{~cm}$ ). 
On note aussi des variations de concentration de la solution absorbée : en débit libre la concentration varie peu $\left(3,18 \mathrm{~g} \cdot \mathrm{I}^{-1}\right.$ en moyenne, pour une solution initiale de $\left.3 \mathrm{~g} \cdot \mathrm{I}^{-1}\right)$; en débit imposé, la solution absorbée est beaucoup plus concentrée $\left(7,1 \mathrm{~g} \cdot \mathrm{I}^{-1}\right)$ que la solution inifiale $\left(3 \mathrm{~g} \cdot \mathrm{I}^{-1}\right)$.

\section{Pression.}

Quelle que soit la série expérimentale, la motricité est faible lorsque la pression basale est supérieure à $13 \mathrm{~cm}$ d'eau.

- En débit imposé, cette pression basale s'accroît rapidement (35 mn environ) de manière irréversible et supprime la motricité.

- En débit libre, les contractions gardent une réelle efficacité propulsive pendant un temps beaucoup plus long ( 1 h 30 à 2 h). La pression basale reste stable, inférieure à $13 \mathrm{~cm}$, la motricité est excellente.

\section{Discussion.}

Les résultats font apparaître que l'intégrité de la motricité est un facteur essentiel pour le rendement de l'absorption, en particulier celle de l'eau et du glucose. Un des facteurs en cause pourrait être la pression intraluminale moyenne, qui s'accroît relativement tôt de manière irréversible en débit imposé alors qu'elle reste relativement stable en débit libre.

Un des résultats fonctionnels de la motricité est la répartition des pressions hydrostatiques autour d'une valeur moyenne, qui correspond au tonus. Ceci rejoint des travaux récents effectués sur l'Homme et sur l'Animal (Binder, 1975 ; Mirkivitch, Menge et Robinson, 1974) qui mettent l'accent sur le rôle joué par la pression hydrostatique dans les mouvements liquidiens.

Nos résultats suggèrent l'importance des conditions mécaniques dans le rendement de l'absorption intestinale.

Commission CNERNA Digestion-Absorption, Tours, 13 novembre 1976.

\section{Références}

BINDER H.-J., 1975, lon transport in large and small intestine. 247-258. In M. H. F. FRIEDMAN. Functions of the stomach and intestine. HM M. Med. Sci. Publ.

DE LAVERNHE-LEMAIRE M.-C., 1976. Influences réciproques de la motricité sur l'absorption du glucose. Etude expérimentale chef le rat. Déductions chez l'homme. D.E.R.B.H. Fac. Méd., Tours.

MIRKIVITCH V., MENGE H., ROBINSON J. W. L., 1974. The effect of intraluminal hydrostatic pressure on intestinal absorption in vivo. Experientia, 30, 912.

THOUVENOT J., ROUGEREAU A., 1964. Possibilités de relation entre les activités absorbantes et contractiles de l'intestin grêle. Etude in vitro chez le rat. C. R. Soc. Biol., 158, 805-809.

ROUGEREAU A., THOUVENOT J., 1965. Effet de variations périodiques de tension longitudinale sur la contractilité ef le tonus du muscle lisse intestinal. Etude in vitro chez le rat. C. $R$. Soc. Biol., 159, 2467.

THOUVENOT J., ROUGEREAU A., FRETTE D., 1966. Réactions de l'intestin isolé de rat à des variations périodiques de tension. Mise en évidence de caractéristiques fonctionnelles. J. Physiol., 158, 269-270. 